\title{
Paravertebral muscles metabolism changes with age in agreement with EMG particularly at L4-5 : a Near Infrared Spectroscopy evaluation (NIRS)
}

Barbara Piovanelli 1 , Massimiliano Gobbo 2 , Sabrina Donzelli 3 , Fabio Zaina 3 , Stefano Negrini 12

1. IRCCS Fondazione Don Carlo Gnocchi, Milano, Milano, ITALY, Italy

2. University of Brescia, Clinical and Experimental Sciences Department, Brescia, Italy

3. ISICO, Italian Scientific Spine Institute, Milan, Italy, Italy

INTRODUCTION: The metabolic changes that occur over time in paravertebral muscles are not yet wellcharacterized. The aim of this study was to test a non-invasive method for the evaluation of the paravertebral lumbar muscles, since there is evidence of a correlation between highly fatigable back muscles and chronic low back pain. ${ }^{1}$

METHODS: We recruited a convenience sample of 37 healthy subjects (18-80 yrs) (20 males and 17 females), to have at least 5 participants per age group ( 15 below 30, 5 aged 30-49, 7 aged 50-59, and 5 aged 60-69 and 70-79). All patients reported no episodes of back pain in the last 5 years. The Biering Sorensen Muscle Test (BSME) was used to cause fatigue: it was repeated three times, to limit adaptation while allowing muscle warm-up. Muscular metabolism of paravertebral muscles was studied checking blood deoxygenation using two Near Infrared Spectroscopy (NIRS) ${ }^{2}$ at the L2-3, L3-4 and L4-5 levels. According to the literature, we used the only reliable phase of the hemoglobin deoxygenated curve ( $\mathrm{HHb})$ that is the rapid one, corresponding to the growth of oxygen consumption before reaching a fully aerobic steady state. We compared all parameters among groups and searched correlations with age, weight, height and BMI. Muscle contraction was studied with surface EMG, particullary Slope Coefficient of Mean Frequency (MNF) was used to analyze EMG Data.

RESULTS: We did not find any difference in the results for the examined levels, even if L2-3 showed to be the most reliable ( $82 \%$ of evaluable curves versus $L 4=76 \%$ and $L 5=71 \%$ ). We did not find any difference for sex or side neither for NIRS nor for EMG. While EMG slope decreased with age, at the L4-5 level the NIRS angle of slope decreased and in men the time to reach plateau increased. We found a direct correlation between EMG and NIRS slope only, but after averaging the two sides. EMG slope was also directly correlated with height, but only in males. We did not find correlations with weight and BMI.

DISCUSSION: With age the growth of EMG signals decreases, and consensually the time to reach an aerobic steady state increases, while the angle of the growth phase consequently decreases. A correlation between metabolic and electromyographic activity has already been demonstrated in low back pain patients, but using a dynamic test and with older, less reliable instruments ${ }^{3}$. Even if the phenomena are clearly physiologically linked, there are other factors to be understood that could play a role (like vascularization, oxygen consumption capacity, etc. $)^{4}$. While L3 is the most used level for NIRS studies, we found correlations only with L4-5 level, where the analysis of rapid phase of the hemoglobin deoxygenated $(\mathrm{HHb})$ was correlated with age, but not to anthropometric parameters.

1. Russo, M. et al. Muscle Control and Non-specific Chronic Low Back Pain. Neuromodulation J. Int. Neuromodulation Soc. 21, 1-9(2018)

2. Ferrari, M. et al.The use of near-infrared spectroscopy in understanding skeletal muscle physiology: recent developments. Philos. Transact. A Math. Phys. Eng. Sci. 369, 4577-4590(2011)

3. Kovacs, KM. et al. Localized oxygen use of healthy and low back pain individuals during controlled trunk movements. J Spinal Disord. Apr;14(2):150-8 (2001)

4. Kalichman, L. et al. The Association between Imaging Parameters of the Paraspinal Muscles, Spinal Degeneration, and Low Back Pain. BioMed Res. Int. 2017, (2017) 